\title{
The miR-1906 mimic attenuates bone loss in osteoporosis by down-regulating the TLR4/MyD88/NF- $\kappa$ B pathway
}

\author{
H. XIE, L. CAO, L. YE, G. SHAN and W. SONG* \\ Department of Rehabilitation, Xuanwu Hospital, Capital Medical University, Beijing, 100053, China
}

Received: March 12, $2020 \bullet$ Accepted: June 12, 2020

Published online: January 06, 2021

(c) 2020 Akadémiai Kiadó, Budapest

\begin{abstract}
In this study, the ability of microRNA-1906 (miR-1906) to attenuate bone loss in osteoporosis was evaluated by measuring the effects of a miR-1906 mimic and inhibitor on the cellular toxicity and cell viability of MC3T3-E1 cells. Bone marrow-derived macrophage (BMM) cells were isolated from female mice, and tartrate-resistant acid phosphatase signalling was performed in miR-1906 mimic-treated, receptor-activated nuclear factor kappa-B (NF- $\kappa \mathrm{B}$ ) ligand (RANKL)-induced osteoclasts. In-vivo, osteoporosis was induced by ovariectomy (OVX). Rats were treated with $500 \mathrm{nmol} / \mathrm{kg}$ of the miR-1906 mimic via intrathecal administration for 10 consecutive days following surgery. The effect of the miR-1906 mimic on bone mineral density (BMD) in OVX rats was observed in the whole body, lumbar vertebrae and femur. Levels of biochemical parameters and cytokines in the serum of miR-1906 mimic-treated OVX rats were analysed. The mRNA expression of toll-like receptor 4 (TLR4), myeloid differentiation primary response 88 (MyD88), p-38 and NF- $\kappa \mathrm{B}$ in tibias of osteoporotic rats (induced by ovariectomy) was observed using quantitative reverse-transcription polymerase chain reaction. Treatment with the miR-1906 mimic reduced cellular toxicity and enhanced the cell viability of MC3T3-E1 cells. Furthermore, osteoclastogenesis in miR-1906 mimic-treated, RANKL-induced osteoclast cells was reduced, whereas the BMD in the miR-1906 mimic-treated group was higher than in the OVX group of rats. Treatment with the miR-1906 mimic also increased levels of biochemical parameters and cytokines in the serum of ovariectomised rats. Finally, mRNA expression levels of TLR4, MyD88, p-38 and NF- $\kappa$ B were lower in the tibias of miR-1906 mimic-treated rats than in those of OVX rats. In conclusion, the miR1906 mimic reduces bone loss in rats with ovariectomy-induced osteoporosis by regulating the TLR4/ MyD88/NF- $\kappa$ B pathway.
\end{abstract}

\footnotetext{
*Corresponding author. E-mail: songwq66@126.com; Tel./fax: +861063012835
} 


\section{KEYWORDS}

miR-1906 mimic, osteoporosis, osteoclast, ovariectomy, inflammation

\section{INTRODUCTION}

Osteoporosis is a bone disease that involves reduced bone density and strength, which, in turn, increases an individual's risk of suffering bone fractures [1]. Osteoporosis is caused by an imbalance between bone resorption and formation. Several factors can contribute to this imbalance, including oestrogen deficiency [2]. Oestrogen deficiency is one reason why the global prevalence of osteoporosis is higher in postmenopausal women [3].

Oestrogen deficiency contributes to the development of osteoporosis because it increases cytokine levels and the activity of the immune system, which can increase osteoclast activity in the body [4]. By altering the activity of osteoclasts and osteoblasts, oestrogen deficiency makes bones more fragile by promoting their remodelling [5]. Current literature suggests that it is the alteration of the toll-like receptor 4/myeloid differentiation primary response 88 /nuclear factor kappa B (TLR4/MyD88/NF- $\kappa$ B) pathway that causes the induction of bone remodelling under oestrogen-deficient conditions [6], and that monkey TLR4 increases cytokine levels, which contributes to the development of an inflammatory response that includes osteoporosis [7]. Oestrogen deficiency also increases oxidative stress levels, which can likewise result in the abovementioned activity [8].

Although several drugs can help manage osteoporosis, including hormone replacement therapy, they are associated with certain risks, including cardiovascular risks and the risk of developing endometrial cancer [8]. Therefore, a new therapeutic approach is needed to manage osteoporosis.

Small and non-coding RNAs, known as microRNAs (miRs) are involved in the development and progression of osteoporosis. They can also alter the activity of osteoclasts and osteoblasts [9]. Several clinical and pre-clinical studies found that miRs alter the bone remodelling process by affecting bone resorption, osteoclastogenic activity and bone formation. Furthermore, a miR1906 mimic was found to enhance neuronal cells and reduce neuropathic pain by regulating the TLR-4/NF- $\kappa$ B pathway [10]. It was also found to be involved in the management of cerebral stroke [11]. Considering the involvement of miR-1906 in these processes, the ability of miR1906 to attenuate bone loss in osteoporosis was evaluated in this study.

\section{MATERIAL AND METHODS}

\section{Cell line and culture}

MC3T3-E1 cells were procured from Peking Union Medical College in Beijing, China, and incubated in penicillin $(100 \mathrm{U} / \mathrm{mL})$, streptomycin $(100 \mathrm{U} / \mathrm{mL})$ and $10 \%$ foetal bovine serum (FBS) supplemented with Dulbecco's modified Eagle medium at $37^{\circ} \mathrm{C}$. The tibias and femurs of C57BL/6J mice were used to extract bone marrow cells. Additional bone marrow-derived macrophages were generated by incubating the isolated cells for 3 days in penicillin $(100 \mathrm{U} / \mathrm{mL})$, streptomycin $(100 \mathrm{U} / \mathrm{mL})$ and 10\% FBS supplemented with $\alpha$-modified Eagle medium. MC3T3- 
E1 cells were then transfected with a miR-1906 mimic or inhibitor for 2 days using Lipofectamine 2000 to promote or inhibit the expression of miR-1906.

Chemicals. The miR-1906 mimic and miR-1906 inhibitor were purchased from Sigma Aldrich (St. Louis, MO, USA). Enzyme-linked immunosorbent assay (ELISA) kits were purchased from R\&D Systems (Minneapolis, MN, USA). Primers used for quantitative reverse-transcription polymerase chain reaction (qRT-PCR) were obtained from Thermo Fisher Scientific (Wilmington, DE, USA).

Cell-Counting Kit-8 (CCK-8) assay. MC3T3-E1 cells $\left(5 \times 10^{3}\right.$ cells/well) were seeded into 12well plates. Each well was treated with $10 \mathrm{ml}$ of CCK- 8 reagent and incubated for $2 \mathrm{~h}$. A microplate reader was used to determine the absorbance at a wavelength of $450 \mathrm{~nm}$. A lactate dehydrogenase $(\mathrm{LDH})$ assay kit was used to estimate cellular toxicity according to the manufacturer's protocol.

\section{Tartrate-resistant acid phosphatase (TRAP) staining}

Bone marrow-derived macrophages $(B M M s)\left(1 \times 10^{5}\right.$ cells $\left./ \mathrm{mL}\right)$ were seeded into a 12 -well plate and treated with $100 \mathrm{ng} / \mathrm{mL}$ of the RANKL and $50 \mathrm{ng} / \mathrm{mL}$ macrophage colony-stimulating factor (M-CSF) for 4 days. The cells were then treated with the miR-1906 mimic on different days, such as days 1, 2 and 3. Cells were fixed on day 4 and stained with tartrate-resistant acid phosphatase (TRAP). An inverted microscope was used to record images.

Animals. Thirty female Sprague-Dawley rats weighing 300-320 g were kept under a 12-h light/ 12 -h dark cycle (humidity, $60 \pm 5 \%$; temperature, $24 \pm 3^{\circ} \mathrm{C}$ ). The protocols used in the animal experiments were approved by the Institutional Animal Ethical Committee of Capital Medical University, Beijing, China (IAEC/CMU/2019/08).

Induction of osteoporosis and treatment. Bilateral ovariectomies were performed in accordance with a previously reported study [12]. All animals were anaesthetised. An incision of approximately $2 \mathrm{~cm}$ was made on the abdomen of each animal. Ovaries were exposed using artery forceps. Bilateral ovaries were removed in each animal, the abdominal fat pad was repositioned and sutures were used to close the wound. Animals in the control group were subjected to the same surgery, but without removal of the ovaries.

The miR-1906 mimic was purchased from Sigma Aldrich (catalogue no. MLMIR0883) and delivered using the MaxSuppressor In Vivo RNA-LANCEr II RNA delivery system in accordance with the manufacturer's protocol. All animals were separated into three different groups - a control group, an OVX (ovariectomy) group and a miR-1906 mimic group. The miR-1906 mimic group received $500 \mathrm{nmol} / \mathrm{kg}$ of the mimic intrathecally for 10 consecutive days following surgery.

Bone mineral density (BMD) measurement. Dual energy X-ray absorptiometry was used to determine the BMD in animals. A Zoletil-Rompun mixture was used to anaesthetise the animals, and scans of the femur, lumbar vertebrae and whole body were used to calculate BMD.

Determination of biochemical parameters. All animals were anaesthetised, and blood was withdrawn from the retro orbital plexuses of the rats. The blood was centrifuged at 2,000 RPM for $10 \mathrm{~min}$ to separate the serum. Levels of osteocalcin (OC), C-telopeptide of type 1 collagen 
(CTX), bone-specific alkaline phosphatase (BSAP), interleukin (IL)-1 $\beta$, tumour necrosis factor (TNF)- $\alpha$ and IL- 6 were measured in the serum of ovariectomised rats using ELISA in accordance with the manufacturer's protocol.

Histopathological analysis. The tibias were isolated from each animal, fixed in $10 \%$ formalin solution and stored at a temperature of $40{ }^{\circ} \mathrm{C}$ for 2 days. Ethylene diamine tetra acetic acid (10\%) was used to decalcify the tibias. Each tibia was then embedded in liquid paraffin to prepare the wax blocks. Tibial tissue sections of $4-\mu \mathrm{m}$ thickness were prepared using a microtome. Haematoxylin and eosin (H\&E) staining was used to stain sections, and a leucocyte acid phosphatase assay kit was used to determine TRAP activity in osteoclasts according to the manufacturer's protocol.

qRT-PCR. TRIzol reagent was used to isolate the total RNA from tibial tissues according to the manufacturer's protocol. A reverse-transcription kit was used to reverse transcribe cDNA from RNA according to the manufacturer's protocol. The ABI Prism 7500 system was then used to analyse samples treated with the SYBR green/fluorescein qPCR Master Mix kit. The conditions were as follows: $50{ }^{\circ} \mathrm{C}$ for $2 \mathrm{~min}$; $95^{\circ} \mathrm{C}$ for $10 \mathrm{~min}$; and 40 cycles of $95^{\circ} \mathrm{C}$ for $30 \mathrm{~s}$ and $60^{\circ} \mathrm{C}$ for 30 s. The resulting data were analysed using the comparative $\mathrm{Ct}$ method. Primers used in this study are listed below:

\begin{tabular}{|c|c|c|}
\hline \multirow[t]{2}{*}{ TLR4 } & Forward & $5^{\prime}$-GCC GGA AAG TTA TTG TGG TGG T-3' \\
\hline & Reverse & 5'-ATG GGT TTT AGG CGC AGA GTT T-3' \\
\hline \multirow[t]{2}{*}{ MyD88 } & Forward & $5^{\prime}$-CAA CCA GCA GAA ACA GGA GTC T-3' \\
\hline & Reverse & $5^{\prime}$-ATT GGG GCA GTA GCA GAT GAA G-3' \\
\hline \multirow[t]{2}{*}{$\mathrm{NF}-\kappa \mathrm{B}$} & Forward & 5'-GCG CAT CCA GAC CAA CAA TAA C-3' \\
\hline & Reverse & 5'-GCC GAA GCT GCA TGG ACA CT-3' \\
\hline \multirow[t]{2}{*}{ p-38 } & Forward & 5'-GAGCGTTACCAGAACCTGTCTC-3' \\
\hline & Reverse & 5'-AGTAACCGCAGTTCTCTGTAGGT-3' \\
\hline \multirow[t]{2}{*}{$\beta$-actin } & Forward & 5'-GCC ATG TAC GTA GCC ATC CA-3', \\
\hline & Reverse & $5^{\prime}$-GAA CCG CTC ATT GCC GAT AG-3' \\
\hline
\end{tabular}

\section{Statistical analysis}

All data are expressed as the means \pm standard error of the mean $(n=10)$. Data were compared using a one-way analysis of variance. Post hoc comparisons were made using Dunnett's test with GraphPad Prism software (ver. 6.1; GraphPad Software, San Diego, CA, USA). In all analyses, $P<0.05$ was considered to denote statistical significance.

\section{RESULTS}

\section{Effects of miR-1906 on cell viability and toxicity}

The effects of the miR-1906 mimic and inhibitor on the cellular toxicity and cell viability of MC3T3-E1 cells are shown in Fig. 1. Treatment with the miR-1906 mimic enhanced cell viability compared to the control group, whereas treatment with miR-1906 inhibitor decreased the viability of MC3T3-E1 cells compared to the control group. 


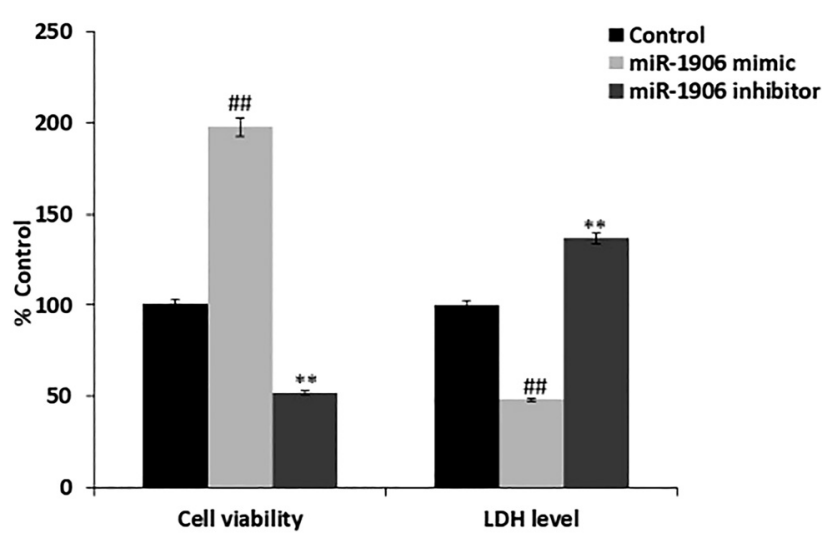

Fig. 1. Effects of the miR-1906 mimic and inhibitor on the cellular toxicity and cell viability of MC3T3-E1 cells. The Cell-counting Kit-8 assay and lactate dehydrogenase (LDH) assays were used to measure cell viability and LDH levels in MC3T3-E1 cells, respectively. Data are represented as the means \pm standard error of the mean (SEM; $n=10$ ); ${ }^{\# \#} P<0.01$ compared to control; ${ }^{* *} P<0.01$ compared to miR-1906 mimic

The effects of the miR-1906 mimic and inhibitor on cellular toxicity were estimated by comparing the level of $\mathrm{LDH}$ in treated samples to that in the control group. There was less LDH in the miR-1906 mimic-treated group than in the control group. However, the miR-1906 inhibitor increased the LDH level in MC3T3-E1 cells compared to the control group. These results show that the miR-1906 mimic increased the proliferation of osteoblasts and reduced the activity of $\mathrm{LDH}$.

Effect of miR-1906 on RANKL-induced osteoclast differentiation. The effects of the miR-1906 mimic were observed on a number of TRAP-positive multinucleated osteoclasts and RANKLinduced osteoclasts in BMM-isolated cells. Treatment with the miR-1906 mimic significantly inhibited osteoclastogenesis. The effect was observed for 3 days. In the miR-1906 mimic group, osteoclastogenesis was inhibited successively for all 3 days of treatment (Fig. 2). Treatment with the miR-1906 mimic reduced the differentiation of osteoclasts and thereby inhibited osteoclastogenesis in osteoclasts with RANKL-induced activation of RANK.

Effect of miR-1906 mimic on bone mineral density. BMD was measured in the whole body, lumbar vertebrae and femurs of miR-1906 mimic-treated osteoporotic rats (Fig. 3). BMD was

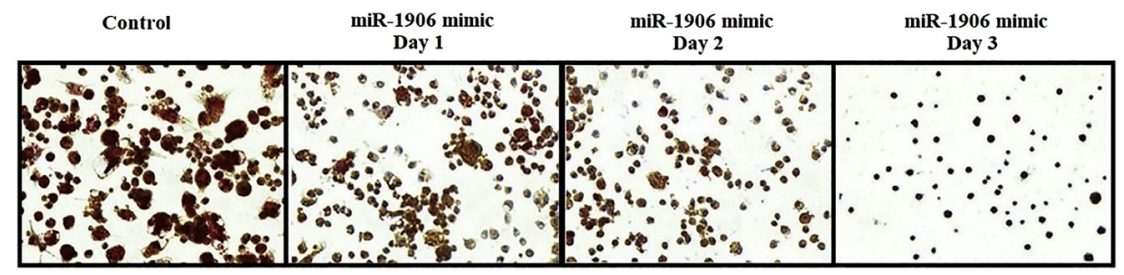

Fig. 2. Effects of the miR-1906 mimic on receptor-activated nuclear factor kappa-B ligand-induced osteoclasts in bone marrow-derived macrophage-isolated cells as visualised using TRAP staining 


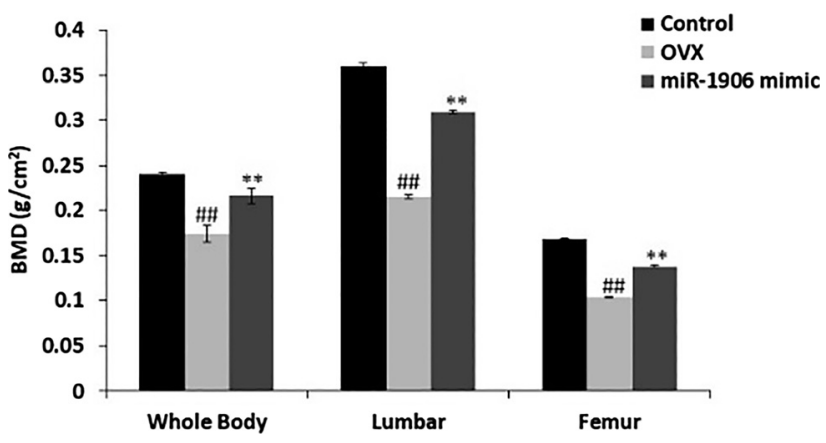

Fig. 3. Effects of the miR-1906 mimic on bone mineral density (BMD) in the whole body, lumbar vertebrae and femurs of rats with ovariectomy-induced osteoporosis (OVX). Dual energy X-ray absorptiometry was used to measure BMD in samples. Data are represented as the means $\pm \operatorname{SEM}(n=10) ;{ }^{\# \#} P<0.01$ compared to control group; ${ }^{* *} P<0.01$ compared to OVX group

Table 1. The miR-1906 mimic enhances osteocalcin (OC), C-telopeptide of type 1 collagen (CTX), procollagen type I N-terminal propeptide (PINP) and bone-specific alkaline phosphatase (BSAP) levels in the serum of rats with osteoporosis induced by ovariectomy (OVX)

\begin{tabular}{lccccc}
\hline Sr. No. & Group & OC $(\mathrm{pg} / \mathrm{mL})$ & CTX $(\mathrm{ng} / \mathrm{mL})$ & PINP $(\mathrm{ng} / \mathrm{mL})$ & BSAP (U/L) \\
\hline 1. & Control & $9.54 \pm 0.62$ & $36.24 \pm 2.1$ & $5.92 \pm 0.65$ & $8.27 \pm 0.41$ \\
2. & OVX & $3.16 \pm 0.24^{\# \#}$ & $146 \pm 8.06^{\# \#}$ & $24.16 \pm 2.34^{\# \#}$ & $14.28 \pm 0.56^{\# \#}$ \\
3. & miR-1906 mimic & $7.02 \pm 0.51^{* *}$ & $53.63 \pm 3.51^{* *}$ & $9.26 \pm 0.96^{* *}$ & $9.25 \pm 0.61^{* *}$ \\
\hline
\end{tabular}

Data are represented as the means \pm standard error of the mean (SEM; $n=10) ;{ }^{\# \#} P<0.01$ compared to control group; ${ }^{* *} P<0.01$ compared to OVX group.

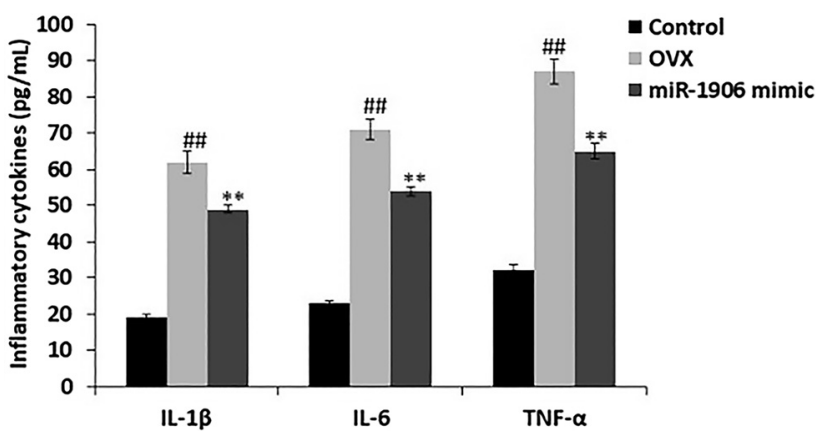

Fig. 4. Effects of the miR-1906 mimic on inflammatory mediators in the serum of osteoporotic rats. Enzyme-linked immunosorbent assay was used to measure levels of inflammatory mediators. Data are represented as the means $\pm \operatorname{SEM}(n=10) ;{ }^{\# \#} P<0.01$ compared to control group; ${ }^{* *} P<0.01$ compared to OVX group 
lower in the whole body, lumbar vertebrae and femurs of the OVX rats compared to the control rats. However, BMD increased following treatment with the miR-1906 mimic in osteoporotic rats. BMD was higher in the whole body, lumbar vertebrae and femurs of miR-1906 mimictreated osteoporotic rats, which suggests that treatment with a miR-1906 mimic prevents osteoporosis.

Effect of miR-1906 mimic on biochemical parameters. The effects of the miR-1906 mimic were observed by analysing the OC, CTX, procollagen type I N-terminal propeptide (PINP) and BSAP levels in the serum of osteoporotic rats. Serum CTX and PINP levels were higher in the OVX group compared to the control group. However, the serum OC level was lower in the OVX

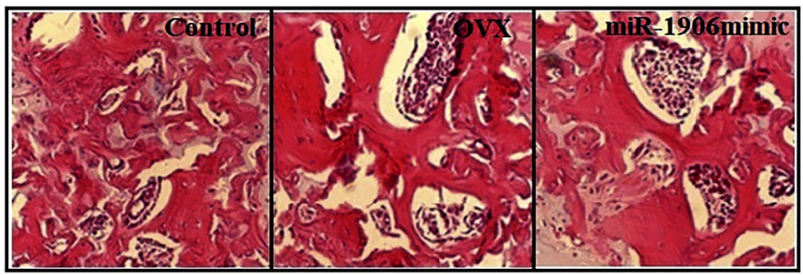

A
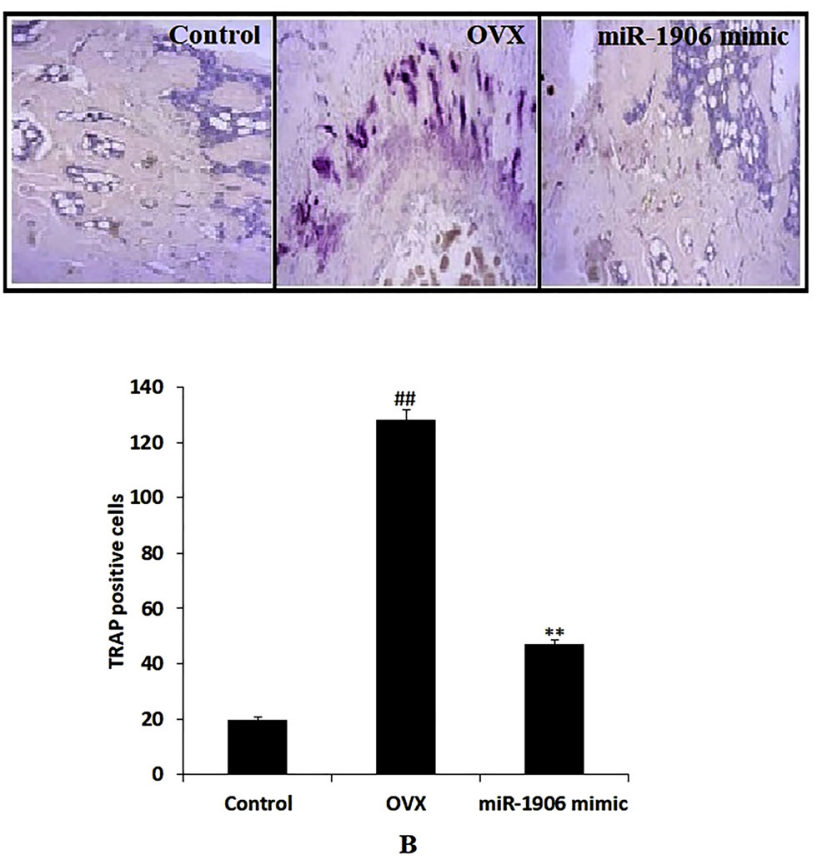

Fig. 5. Effects of the miR-1906 mimic on the tibial tissue of rats with osteoporosis induced by ovariectomy. (A) Haematoxylin and eosin staining of tibial tissue section. (B) The percentage of TRAP-stained positive cells in the section of tibial tissue was calculated using a leucocyte acid phosphatase assay kit. Data are represented as the means $\pm \operatorname{SEM}(n=10) ;{ }^{\# \#} P<0.01$ compared to control group; ${ }^{* *} P<0.01$ compared to OVX group 
group than in the control group. Furthermore, serum BSAP activity was higher in the OVX group than in the control group. Treatment with the miR-1906 mimic changed the levels of OC, CTX, PINP and BSAP in the serum of osteoporotic rats (Table 1). The altered levels of the biochemical parameters suggest that treatment with the miR-1906 mimic protects against osteoporosis.

The miR-1906 mimic attenuates inflammatory mediators. The levels of inflammatory mediators were measured in the serum of miR-1906 mimic-treated osteoporotic rats. The serum levels of IL-1 $\beta$, IL- 6 and TNF- $\alpha$ were significantly enhanced in the OVX group compared to the control group. There were less inflammatory cytokines in the serum of miR-1906 mimic-treated rats compared to OVX rats (Fig. 4).

The miR-1906 mimic ameliorates bone resorption. The effect of miR-1906 mimic treatment on bone resorption was examined via histopathological analysis of the tibial tissue of osteoporotic rats using $\mathrm{H} \& \mathrm{E}$ and TRAP staining (Fig. 5A and B). In the OVX group, metaplasia of tibia cells with cancellous bone was observed. In addition, the bone marrow space was larger compared to that in rats in the control group. Treatment with the miR-1906 mimic reversed the altered pathophysiology of tibial tissue in osteoporotic rats (Fig. 5A). Furthermore, the number of TRAP-positive multinucleated osteoclasts was higher in the bone tissue of rats in the OVX group than in the control group. There were also fewer TRAP-positive multinucleated osteoclasts in the tibial tissue of rats treated with the miR-1906 mimic than in the tissue of rats in the OVX group (Fig. 5B).

The miR-1906 mimic ameliorates the mRNA expression of TLR4, MyD88, p-38 and NF-KB proteins. The mRNA expression of TLR4, MyD88, p-38 and NF- $\kappa$ B was estimated in the tibial tissues of miR-1906 mimic-treated osteoporotic rats (Fig. 6). There was an increase in the mRNA expression of TLR4, MyD88, p-38 and NF- $\kappa \mathrm{B}$ in the tibial tissue of rats in the OVX group compared to rats in the control group. Treatment with the miR-1906 mimic lowered the mRNA expression of TLR4, MyD88, p-38 and NF- $\kappa$ B in the tibial tissue of osteoporotic rats.

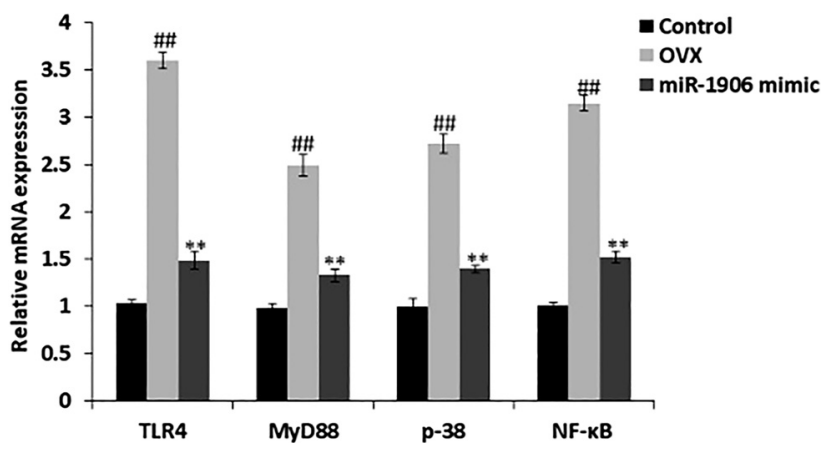

Fig. 6. Effects of the miR-1906 mimic on the mRNA expression of TLR4, MyD88, p-38 and NF- $\kappa$ B in the tibias of osteoporotic rats. Levels of mRNA expression were measured using quantitative reverse-transcription polymerase chain reaction. Data are represented as the means \pm SEM $(n=10) ;{ }^{\# \#} P<0.01$ compared to control group; ${ }^{* *} P<0.01$ compared to OVX group 


\section{DISCUSSION}

Osteoporosis, a bone mass disorder, commonly occurs in women following menopause and increases the chances of developing bone fractures [13]. Conventional drugs available for the management of osteoporosis have significant limitations. Therefore, there is a need to develop new therapy to help manage osteoporosis.

In this study, the ability of a miR-1906 mimic to attenuate bone loss in an osteoporotic rat model induced by ovariectomy was evaluated. The effects of the miR-1906 mimic and inhibitor on the cellular toxicity and cell viability of MC3T3-E1 cells were investigated. BMM cells were isolated from female mice, and TRAP signalling was performed in miR-1906 mimic-treated, RANKLinduced osteoclasts. BMD was estimated in the whole body, lumbar vertebrae and femurs of miR1906 mimic-treated OVX rats. Levels of biochemical parameters and cytokines were analysed in the serum of miR-1906-mimic-treated OVX rats. The mRNA expression of TLR4, MyD88, p-38 and NF- $\kappa \mathrm{B}$ in the tibias of the osteoporotic rats was analysed using qRT-PCR.

The effects of miR-1906 on cellular toxicity and viability were also observed in pre-osteoblast cells-the MC3T3-E1 cells. Treatment with the miR-1906 mimic enhanced cell viability, and cell toxicity was less pronounced compared to the control cells. The activity, survival and differentiation of osteoclasts are regulated by cytokines such as M-CSF and RANKL, which are expressed by osteoblast cells [14]. In this study, treatment with a miR-1906 mimic reduced osteoclastogenesis in RANKL-induced osteoclasts derived from BMM cells.

There are several biomarkers of bone formation and resorption, including the activities of CTX, OC, BSAP and PINP [15]. Treatments used for the management of osteoporosis attenuate the altered levels of these biomarkers. Likewise, treatment with a miR-1906 mimic attenuated altered levels of CTX, OC, BSAP and PINP in the serum of ovariectomised rats. Inflammatory cytokines have also been reported to regulate bone resorption. Furthermore, a deficiency of oestrogen results in an increase in the level of inflammatory cytokines [16]. According to the findings of this study, miR-1906 mimic treatment reduced the levels of inflammatory cytokines significantly $(P<0.01)$ compared to levels in the OVX group.

TLR4 activation is also involved in several other pathological conditions such as weight gain, insulin resistance, inflammation and osteoclastogenesis [7]. One study suggests that TLR4 expression increases in the brain tissue of rats with osteoporosis induced by ovariectomy [17]. The data from this study support those findings. MyD88 signalling also contributes to the proliferation and development of osteoblasts [18]. Bone resorption and formation activities were reported to be lower in MyD88 ${ }^{-/-}$mice [19]. Treatment with a miR-1906 mimic ameliorated the altered expression of MyD88 and TLR4 in the tibial tissue of osteoporotic rats.

\section{CONCLUSION}

In conclusion, miR-1906 mimic reduced osteoclastogenesis/bone resorption in a rat model of osteoporosis induced by ovariectomy via regulation of the TLR4/MyD88/NF- $\kappa \mathrm{B}$ pathway. Ultimately, treatment with a miR-1906 mimic attenuates bone loss in osteoporosis.

Conflict of interest: None 


\section{ACKNOWLEDGEMENTS}

The authors of this manuscript thank Xuanwu Hospital and Capital Medical University (China) for providing the facilities necessary for conducting this work.

The English in this document has been checked by at least two professional editors, both native speakers of English. For a certificate, please see: http://www.textcheck.com/certificate/IqZ2w5.

\section{REFERENCES}

1. Office of the Surgeon General (US). Bone health and osteoporosis: a report of the Surgeon General. Rockville (MD): Office of the Surgeon General (US); 2004.

2. Riggs BL. The mechanisms of estrogen regulation of bone resorption. J Clin Invest 2000; 106: 1203-4.

3. Ji M-X, Yu Q. Primary osteoporosis in postmenopausal women. Chronic Dis Transl Med 2015; 1: 9-13.

4. Zhao R. Immune regulation of osteoclast function in postmenopausal osteoporosis: a critical interdisciplinary perspective. Int J Med Sci 2012; 9: 825-32.

5. Khosla S, Oursler MJ, Monroe DG. Estrogen and the skeleton. Trends Endocrinol Metab 2012; 23 : 576-81.

6. Feng X, McDonald JM. Disorders of bone remodeling. Annu Rev Pathol 2011; 6: 121-45.

7. Alonso-Pérez A, Franco-Trepat E, Guillán-Fresco M, Jorge-Mora A, López V, Pino J, et al. Role of toll-like receptor 4 on osteoblast metabolism and function. Front Physiol 2018; 9: 504.

8. Geng Q, Gao H, Yang R, Guo K, Miao D. Pyrroloquinoline quinone prevents estrogen deficiency-Induced osteoporosis by inhibiting oxidative stress and osteocyte senescence. Int J Biol Sci 2019; 15: 58-68.

9. Fröhlich L. MicroRNAs at the interface between osteogenesis and angiogenesis as targets for bone regeneration. Cells 2019; 8: 121.

10. Fang X, Zhou H, Huang S, Liu J. MiR-1906 attenuates neuropathic pain in rats by regulating the TLR4/ mTOR/Akt signaling pathway. Transl Neurosci 2019; 10: 175-9.

11. Xu X, Wen Z, Zhao N, Xu X, Wang F, Gao J, et al. MicroRNA-1906, a novel regulator of toll-like receptor 4, ameliorates ischemic injury after experimental stroke in mice. J Neurosci 2017; 37: 10498-515.

12. Kim JL, Moon CW, Son YS, Kim SJ. Combined effect of bilateral ovariectomy and anterior cruciate ligament transection with medial meniscectomy on the development of osteoarthritis model. Ann Rehabil Med 2016; $40: 583$.

13. Tella SH, Gallagher JC. Prevention and treatment of postmenopausal osteoporosis. J Steroid Biochem Mol Biol 2014; 142: 155-70.

14. Boyce BF. Advances in the regulation of osteoclasts and osteoclast functions. J Dent Res 2013; 92: 860-7.

15. Paul T, Shetty S, Kapoor N, Bondu J, Thomas N. Bone turnover markers: Emerging tool in the management of osteoporosis. Indian J Endocrinol Metab 2016; 20: 846.

16. Weitzmann MN. Estrogen deficiency and bone loss: an inflammatory tale. J Clin Invest 2006; 116: $1186-94$.

17. Mouihate A. TLR4-mediated brain inflammation halts neurogenesis: impact of hormonal replacement therapy. Front Cell Neurosci 2014; 8.

18. Sato N, Takahashi N, Suda K, Nakamura M, Yamaki M, Ninomiya T, et al. MyD88 but not TRIF is essential for osteoclastogenesis induced by lipopolysaccharide, diacyl lipopeptide, and IL-1alpha. J Exp Med 2004; 200: 601-11.

19. Putnam NE, Fulbright LE, Curry JM, Ford CA, Petronglo JR, Hendrix AS, et al. MyD88 and IL-1R signaling drive antibacterial immunity and osteoclast-driven bone loss during Staphylococcus aureus osteomyelitis. PLoS Pathog 2019; 15: e1007744. 